Ann. Zootech., I977, 26 (I), II9-I24.

\title{
Note \\ Variation du rapport poids/longueur des carcasses bovines
}

\author{
B. L. DUMONT \\ Laboratoire de Recherches sur la viande, \\ Centre national de Recherches zootechniques, I.N.R.A., \\ 78350 Jouy-en-Josas (France)
}

\section{Résumé}

On a étudié la variation du rapport $(\mathrm{P} / \mathrm{L})$ du poids de la calcasse $(P)$ à sa longueur $\mathrm{L}$ dans un échantillon de bovins adultes comprenant divers types commerciaux. Le rapport augmente avec le poids selon l'équation

$$
\mathrm{P} / \mathrm{L}=0,0064 \mathrm{P}+0,290 .
$$

Son accroissement va de pair avec une amélioration de la conformation. L'utilisation de ce rapport pour le classement des carcasses est discuté.

L'emploi des relations existant entre la longueur et le poids des carcasses pour caractériser la conformation de ces dernières a été proposé par YEATES (I952). Celui-ci a suggéré de considérer à cet effet la valeur du "fleshing index " qui représente la différence de poids existant entre le poids réel d'une carcasse et le poids théorique "moyen » qu'elle devrait présenter, compte tenu de sa longueur.

Par la suite on a pu envisager, plus simplement, de caractériser la carcasse par la valeur du rapport de son poids $(\mathrm{P})$ à sa longueur (L). On a montré ainsi que le rapport $\mathrm{P} / \mathrm{L}$ augmentait avec le poids de la carcasse et qu'il différait entre les sexes (Dumont et al., I963) et entre les races (Dumont, r969). Ce rapport a été utilisé pour comparer la conformation de divers types de productions (Roux, TEIssier et JourNÉ, I975). On a proposé récemment en Australie d'utiliser les 
relations entre le poids et la longueur pour apprécier la conformation des carcasses en vue de leur classement commercial (anonyme, I976).

La présente note rapporte les résultats obtenus par l'utilisation du rapport du poids à la longueur de la carcasse dans un échantillon comprenant les diverses classes de qualité présentes sur le marché français.

On a considéré 66 bovins comprenant des sujets des quatre types sexuels (génisse, vache, bœuf et mâle entier) et de différentes races (Charollaise, Française frisonne Pie noire, Holstein, Maine-Anjou et Normande). Les animaux ont été sacrifiés dans les abattoirs du C.N.R.Z. Dès la fin des opérations d'abattage et de préparation des carcasses on a relevé le poids de la carcasse chaude $(P$, en $\mathrm{kg})$. Vingt-quatre heures après, la carcasse était mesurée et jugée pour la conformation d'après les propositions faites par de BoER et al. (I974). La longueur du corps (L en $\mathrm{cm}$ ) était mesurée avec un mètre métallique souple, du bord inférieur de la symphyse ischio-pubienne au milieu du bord antérieur de la première côte. On a pratiqué trois mesures de la longueur et retenu la valeur moyenne. La classe générale de conformation des carcasses était appréciée par comparaison aux standards de référence des normes de charnure de la Fédération Européenne de Zootechnie selon la méthode proposée par Dumont, Le Guelte et Sornay (I975).

Dans l'échantillon étudié le poids de carcasse $(349,9 \pm 82, \mathrm{I} \mathrm{kg})$ présentait une variation importante. Le coefficient de variation était de $23,46 \mathrm{p}$. Ioo et l'intervalle de variation allait de $\mathbf{I} 67,4$ à $531,0 \mathrm{~kg}$. La variation de longueur $(\mathbf{I} 37,2$ $\pm 6,8 \mathrm{~cm}$ ) était moindre : le coefficient de variation n'était que de 4,97 p. Ioo et l'intervalle de variation allait seulement de 124,6 à $150,6 \mathrm{~cm}$.

Cette différence apparente dans l'importance de variation entre le poids et la longueur est assez logique dans la mesure où le poids intervient comme le cube d'une dimension linéaire; en considérant la racine cubique du poids à la place du poids, l'intervalle de variation serait ramené de 5,5 I à 8 , Io p. IoO.

$\mathrm{Au}$ fur et à mesure que la carcasse s'alourdit le rapport $\mathrm{P} / \mathrm{L}$ augmente; la relation entre les deux variables est très étroite $(r=+0,982)$ et s'exprime par l'équation :

$$
\mathrm{P} / \mathrm{L}=0,0064 \mathrm{P}+0,290
$$

La très forte liaison enregistrée entre les deux caractères s'explique, non seulement par le fait que la valeur du rapport $\mathrm{P} / \mathrm{L}$ tient compte de la valeur du poids, mais aussi parce qu'il existe une relation assez étroite entre le poids et la longueur de la carcasse $(r=+0,56)$.

L'importance de la liaison positive entre le rapport $\mathbf{P} / \mathrm{L}$, et le poids est du même ordre que celle qui avait été trouvée par Dumont et ARnoux (Ig68) sur le marché de Paris, pour des ensembles de types sexuels considérés séparément (bøuf, génisses et vaches). Au niveau d'une population d'animaux commerciaux la liaison entre le poids et le rapport $\mathrm{P} / \mathrm{L}$ a donc une portée très générale.

En s'alourdissant la carcasse devient plus compacte puisque la charge supportée par l'axe du corps, que définit la ligne déterminant la longueur de la carcasse, augmente. La variation enregistrée dans la population pour le rapport $\mathbf{P} / \mathrm{L} \quad(2,54$ $\pm 0,54$, avec un coefficient de variation de $21,26 \mathrm{p}$. Ioo et un intervalle de 1,33 à $3,68 \mathrm{~kg} / \mathrm{cm}$ ) s'explique essentiellement par la variation de poids des carcasses. 
RAPPORT POIDS/LONGUEUR DES CARCASSES BOVINES

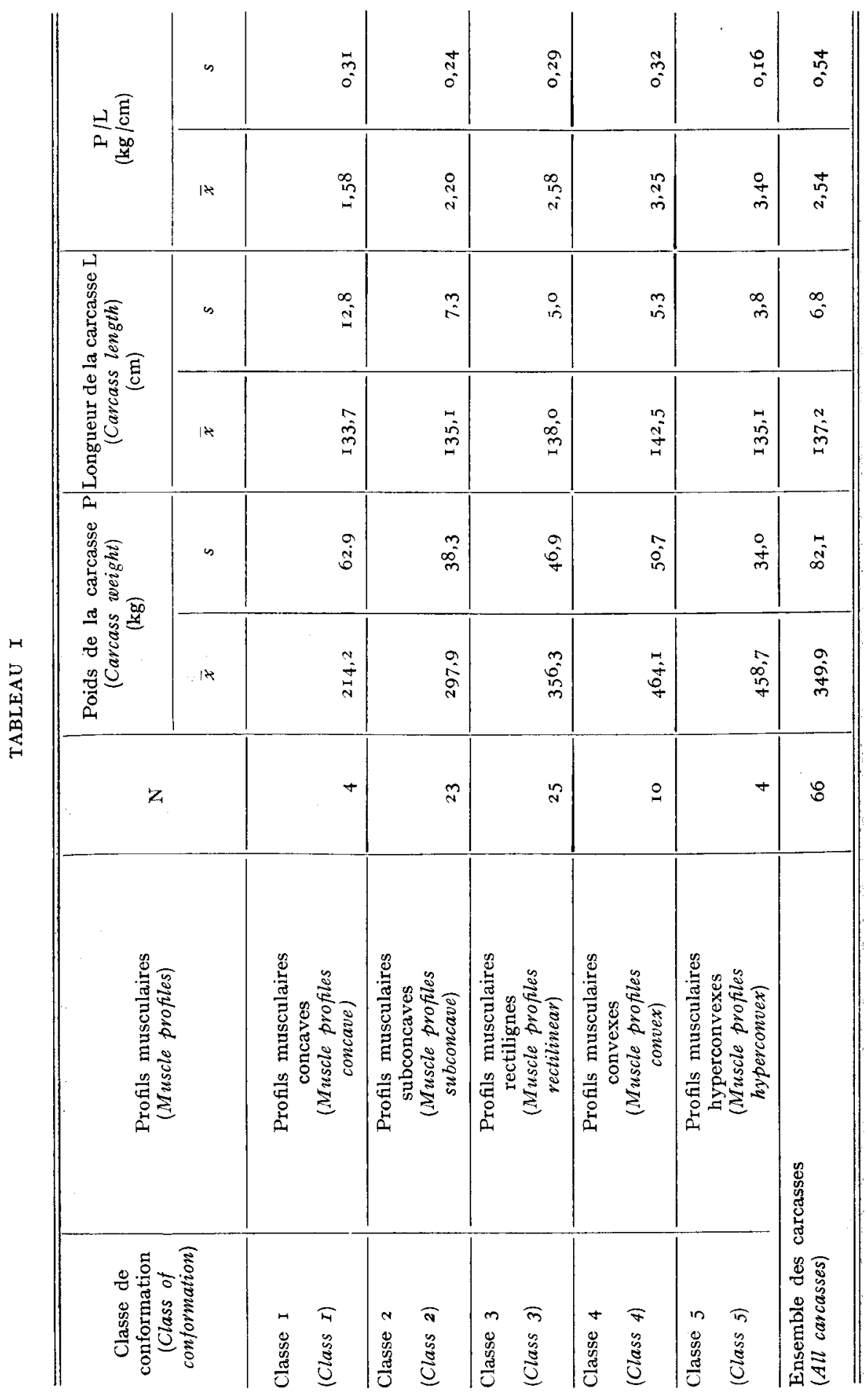




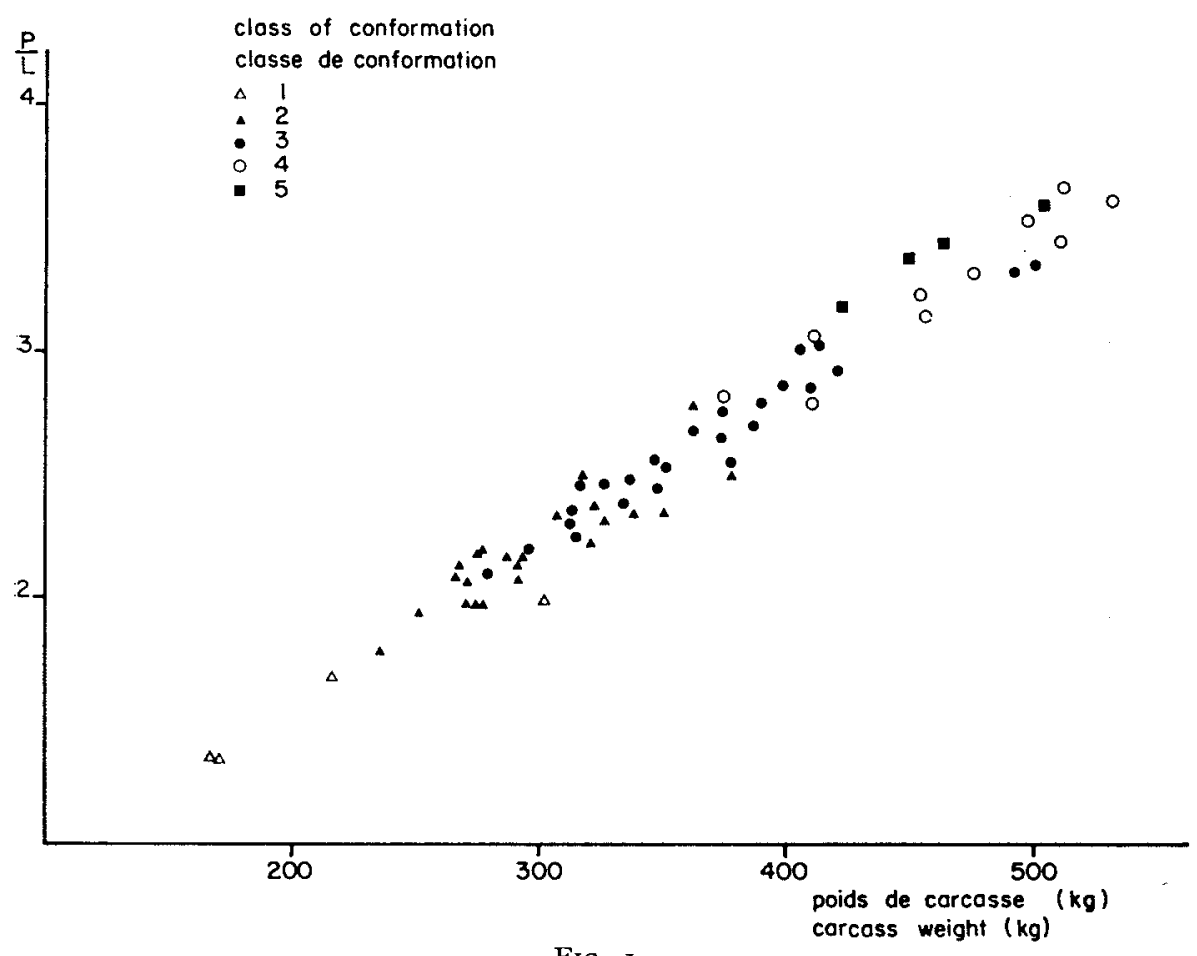

FIG. I.

Une part de la variation (non calculée ici) peut aussi provenir des différences de conformation des animaux à poids équivalent. C'est ainsi que les animaux hypertrophiés présentent, relativement à leur poids, une charge $\mathrm{P} / \mathrm{L}$ plus élevée en raison de leur moindre longueur corporelle (cf. tabl. I, classe $\mathrm{V}$ des profils musculaires hyperconvexes).

Pratiquement l'accroissement du rapport $\mathrm{P} / \mathrm{L}$ avec le poids de carcasse implique que l'on ne procède pas à des comparaisons entre animaux de poids différents sur la base des valeurs brutes de leurs rapports. Lorsqu'on utilise les valeurs du rapport $\mathrm{P} / \mathrm{L}$ pour comparer des compacités de carcasses d'origines différentes et de poids différents, il est nécessaire d'ajuster les valeurs des rapports $P / L$ pour tenir compte, dans les comparaisons, de la part revenant à l'influence du poids dans les différences observées entre carcasses pour ce caractère. L'équation (I) est proposée comme équation de référence pour procéder aux ajustements envisagés. Sur la figure I on a indiqué, avec des symboles appropriés, la classe générale de conformation à laquelle appartenait chaque carcasse. Le tableau I présente, d'autre part, les valeurs moyennes du poids de carcasse $(\mathrm{P})$ et du rapport $\mathrm{P} / \mathrm{L}$ des carcasses de chacune des classes de conformation.

On voit d'après la figure I et le tableau I qu'au niveau des moyennes on assiste, avec l'augmentation corrélative du rapport $\mathrm{P} / \mathrm{L}$, à une amélioration de la conformation. Le coefficient de corrélation entre la valeur du rapport $\mathrm{P} / \mathrm{L}$ et la note de classe de conformation est de $+0,86$. 
Cette tendance - au sein de l'échantillon - à l'amélioration de la conformation en fonction de l'accroissement du poids de la carcasse est à rapprocher de l'observation faite précédemment sur un échantillon plus important de bovins $(\mathrm{N}=208)$ où il avait été constaté qu'avec l'augmentation de la qualité commerciale, en allant de la "fabrication " à l' " extra ", on enregistrait simultanément une augmentation sensible de la note de développement musculaire et une augmentation du poids de la carcasse (Dumont, I967).

On note aussi qu'il existe un certain chevauchement des groupes de conformation entre eux, ce qui amène à penser que la valeur $\mathrm{P} / \mathrm{L}$ n'est pas seule en cause dans la détermination de la classe de conformation. En fait, la variation du rapport $\mathrm{P} / \mathrm{L}$ n'explique que $74 \mathrm{p}$. Ioo de la note de classe de conformation. La compacité massique exprimée par la charge de la carcasse en $\mathrm{kg} / \mathrm{cm}$ de longueur est donc partiellement indépendante de la compacité volumique que traduit la classe de conformation. Ce point devrait être éclairci avant de songer à utiliser la valeur du rapport $\mathrm{P} / \mathrm{L}$ dans le but de comparer les animaux de boucherie entre eux et d'établir, d'après cette valeur, leur classement commercial.

Reģu pour publication en octobre 1976.

\section{Remerciements}

Ce travail a été réalisé avec la collaboration de M. Roy et avec l'aide de M. Morand (de l'I.T.E.B.) que nous tenons à remercier pour leur coopération.

\section{Summary}

\section{Variation in the weight/length ratio of bovine carcasses}

Variation in the weight-to-length ratio (P/L) was studied on a sample of adult carcasses of various commercial types. The ratio $(P$ in $\mathrm{kg}, \mathrm{L}$ in $\mathrm{cm}$ ) increased with carcass weight, according to the relation.

$$
\frac{\mathrm{P}}{\mathrm{L}}=0,0064 \mathrm{P}+0,290 \quad r=+0,982
$$

Use of the weight-to-length ratio for grading carcasses is discussed, in view of the correlation $(y=+0,86)$ found between its value and the carcass score for conformation.

\section{Références bibliographiques}

Anonyme, 1976. Australians test electronic beef grading system. The Natl Prov., 174 (25). 43-44.

De Boer H., Dumont B. L., Pomeroy R. W., Weniger J. H., 1974. Manual on E.A.A.P. Reference methods for the assessment of carcass characteristics in cattle. Livestock Prod. Sci., 1, $15 \mathrm{I}-\mathrm{I} 64$.

Dumont B. L., 1967. Étude sur les qualités de carcasses de bovins en France. Inform. statist. Commun. eur. (4), 72 pp.

Dumont B. L., I969. Influence du type ethnique sur les rapports entre le poids, la longueur et la masse musculaire de la carcasse des bovins. Ann. Génét. Sél. anim., 1 (2), p. I9I. 
Dumont B. L., Arnoux J., I968. Influence de différents caractères de la carcasse de bovins sur la détermination de son prix. Études de statistique agricole. Office statistique des communautés européennes, 1, I-30.

Dumont B. L., Boccard R., Renou Y., Charpentier J., i963. Observations sur les qualités de boucherie de taurillons charollais. Bull. Techn. (I), Lab. Rech. Viande INRA. I3 pp. ronéotypées.

Dumont B. L., Le Guelte P., Sornay J., 1975. Le jugement de la formation des carcasses de bovins. Technipel édit. Paris, $26 \mathrm{pp}$.

Roux M., TeIsSiER J. H., Journé H. E., I975. Influence du mode d'élevage de la naissance à 8 mois sur la croissance, l'efficacité alimentaire et les caractéristiques des carcasses et de la viande de taurillons Pie Rouge de l'Est abattus à I6-17 mois. Ann. Zootech., 24, 57I-578.

Yeates N. T. M., I952. The quantitative definition of cattle carcasses. Aust. J. Agric. Res., 3, 68-94. 